\title{
Radiological Evaluation of Lumbar Instability
}

\author{
${ }^{1}$ Bidhya Bhusan Tamrakar, ${ }^{2}$ Nitin Tandra, ${ }^{3}$ Huang Yonghui, \\ ${ }^{4}$ Li Dapeng, Sun Jifu, \\ ${ }^{1,2,3,4}$ Orthopedics Department No. 2, Affiliated Hospital of Jiangsu University, Zhenjiang, Jiangsu Province, P.
} R. China

\begin{abstract}
Lumbar instability of the lumbar spine is thought to be a possible mechanical mechanism underlying low back pain and is often an important factor in determining surgical indication for spinal fusion and decompression. Instability of the lumbar spine, however, remains a poorly understood subject. Clinical presentation is not specific, and the relationship between radiologic imaging of instability and its symptoms is controversial. Because of its simplicity, low expense, and pervasive availability, functional flexion-extension radiography is the most thoroughly studied and the most widely used method in the imaging diagnosis of lumbar intervertebral instability. In this article, we provide an overview of the current concepts of vertebral instability, focusing on degenerative lumbar intervertebral instability, and review the different imaging modalities most indicated in diagnosing vertebral instability.

The spine is made up of segments, described as "locomotive segments," consisting of two vertebrae and the interconnecting soft tissue. In normal conditions of daily life, the spine is able to meet essential functional requirements: strength, mobility, and stability.

Spinal stability is defined as the ability for the vertebrae to maintain their relationship and limit their relative displacements during physiologic postures and loads. The requirement of stability is essential to the spinal column to prevent premature mechanical and biologic deterioration of its components. It is also fundamental to protect the spinal cord and nerve roots and to minimize energy expenditure.

One important mechanical function of the lumbar spine is to support the upper body by transmitting compressive and shearing forces to the lower body during the performance of everyday activities. To enable the successful transmission of these forces, mechanical stability of the spinal system must be ensured. Stability of the lumbar spine as a whole is maintained by the cooperation of disks, joints, ligaments, and muscles. Degenerative processes in the disk and facet joints affect the stability of the motion segment. Although segmental instability is often used synonymously with degenerative spondylolisthesis, it is clear there are numerous other conditions that are potentially unstable (spinal acute trauma, surgery, spondylolysis, tumors, or infections).
\end{abstract}

Key words: Lumbar instability, degenerative spondylolisthesis, MR imaging

\section{Definition Of Instability}

Despite the effort of several authors to define lumbar spinal instability, no generally accepted definition is yet available ${ }^{[1]}$. A major problem is that the concept of instability means different things to different specialists (clinicians, radiologists, bioengineers). However, a reasonable definition has been proposed by Pope and Panjabi ${ }^{[2]}$ and Frymoyer and Selby ${ }^{[3]}$. By advocating a biomechanical approach, they defined instability as a loss of motion segment stiffness, such that force application to that motion segment produces abnormally great motion compared to that of a normal spine. In other words, instability can be defined as an abnormal response to applied loads characterized kinematically by abnormal movement in the motion segment beyond normal constraints. This abnormal movement can be explained by damage to the restraining structures (ie, facet joints, disks, ligaments, and muscles) that, if damaged or lax, will lend to altered equilibrium and thus instability. In a biomechanical sense, stiffness is defined as the ratio of the load applied to a structure to the resulting motion.

\section{Lumbar Instability}

Upright posture and upright weight bearing in humans cause excess stresses that are maximal at and suprajacent to the lumbosacral junction. This results in more severe age-related changes in these spinal segments. The degenerative processes of the lumbar spine generally initiate from the intervertebral disk, at the level at which progressive biochemical and structural changes take place, leading to a modification in its physical properties of elasticity and mechanical resistance. Disk degeneration, which affects the whole population, is commonly seen from age 30 years onward. The degenerative process in the disk results in a gradual disruption of the collagen fibers and reduction in the proteoglycan contents, with a gradual loss of water contents and elasticity of the disk. More than 50\% of autopsy specimens obtained from individuals in their 3rd and 4th decade of life show peripheral tears of the annulus fibrosus. After age 40 years, the disk becomes 
progressively more fibrous and disorganized due to aging and degeneration; the final stage is represented by regions of amorphous fibrocartilage. This will at some point entail a superoinferior narrowing and eventual collapse of the intervertebral disk.

Three clinically relevant consequences of acquired collapse of the intervertebral disk are (a) pathologic changes in the vertebral bodies, with osteophyte development; $(b)$ anterior bulging of the flaval ligaments and posterior bulging of the posterior longitudinal ligament, with consequential narrowing of the central spinal canal ; and (c) posterior bulging of redundant posterior disk surface, with narrowing of the central spinal canal and of the inferior recesses of the neural foramina. Moreover, intervertebral disk degeneration and acquired collapse permit the adjacent vertebrae to slide back and forth over each other. This results in laxity of the ligamentous network responsible for binding the vertebrae together and leads to craniocaudal partial subluxation of the facet joints, which may be asymmetric from side to side. Subsequent stresses on the facet joints then result in osteoarthritis with ostheophytosis, which in turn causes narrowing of the lateral recesses of the central spinal canal and of the neural foramina. Furthermore, partial subluxation of the facet joints leads to the collision of the apex of the superior articular facet process with the overlying pars interarticularis and pedicle. Continued collision of these structures results in ostheophytosis and consequently to further narrowing of the central spinal canal and of the neural foramina.

Osteoarthritis of the facet joints, which may occur independently of the disk, is characterized by the thinning of the cartilage, sclerotic changes in the subchondral bone, osteophyte formation, synovial inflammation, and capsular ligament laxity. In more severe forms of the process, osteoarthritis of the facet joints may allow hypermobility of the facet joint and then may lead to a spondylolisthesis. This term refers to the forward slippage (by any cause) of a vertebra on the subjacent one in the sagittal plane. In 1930, Junghanns ${ }^{[4]}$ defined lumbar vertebral slippage in the absence of a bone defect in the pars interarticularis as pseudospondylolisthesis. This was later categorized as degenerative lumbar spondylolisthesis by Newman and Stone ${ }^{[5]}$. Backward vertebral slippage, a type of spondylolisthesis, has been called retrolisthesis.

Osteoarthritis of the facet joints, with consequential loss of their normal structural support, plays an important role in the development of degenerative spondylolisthesis. The facet joint orientation of the lower lumbar spine in a normal population and in a population of patients with degenerative spondylolisthesis at L4-5 level was also characterized. At the L4-5 level, a more sagittal orientation of facet joints was found in the degenerative spondylolisthesis group, when compared with the normal group. This sagittal orientation facilitates vertebral slippage when the other predisposing factors are present. Because of these abnormalities and the preponderance of coronal orientation of the L5-S1 facet joints, the majority of degenerative spondylolisthesis occurs at the L4-5 level.

The relationship between lumbar instability and degenerative spondylolisthesis was suggested by Kirkaldy-Willis ${ }^{[6]}$ and Farfan who, in a functional sense, proposed three clinical and biomechanical stages of lumbar spine degenerative changes: temporary dysfunction, unstable phase, and stabilization. Spinal degenerative changes included disk degeneration, facet joints osteoarthritis, ligamentous degeneration, and muscle alterations. The duration of each stage varies greatly, and there are no clear-cut clinical signs or symptoms to distinguish one stage from the next. The first phase, defined as the temporary dysfunction phase, is associated with slight reversible anatomic changes. The second, or unstable, phase is characterized by disk height reduction, ligament and joint capsule laxity, and facet joint degeneration. In the third, or stabilization, phase, osteophytes and marked disk space narrowing lead to stabilization of the motion segment with a reduction (partial or complete) in its range of motion, sometimes after spondylolisthesis has already occurred. On the basis of this model, the radiologic observation of degenerative spondylolisthesis does not necessarily indicate that intervertebral instability is still present at the time of imaging because a new stabilization may have already occurred.

Authors of several biomechanical and clinical studies have reported the association of disk degeneration with segmental instability, confirming Kirkaldy-Willis ${ }^{[6]}$ and Farfan's concept . However, this association was not confirmed in other studies.

To test the validity of this three-stage hypothesis, Axelsson and Karlsson ${ }^{[7]}$ assessed the intervertebral mobility for the two most distal lumbar disk levels in 18 adult patients with low back pain, disk degeneration, and no prior spinal surgery. Each spinal segment was placed in one of five categories according to the grade of disk degeneration. They observed that intervertebral mobility undergoes changes throughout the degenerative process and that a stage of relative stabilization is reached after the degenerative process has reduced the disk height by at least $50 \%$ (category III). Even so, they concluded that absolute stability could not be assumed even for spine segments with greater than $50 \%$ disk height reduction, as some mobility may still persist in such segments.

Segmental lumbar spinal instability is a temporary phase in the degenerative process of the lumbar spine. This process of degeneration has been sub-divided into three phases. 
(a) Dysfunction - is the earliest phase in which the affected level of the lumbar spine does not function normally but pathological changes are minimal.

(b) Instability - intermediate phase in which the disc height is diminished and the annulus fibrosus bulges all around the circumference of the disc, the ligaments and capsule of the posterior facet joint are lax and the articular cartilage is degenerated. This leads to increased and abnormal movement.

(c) Restabilization - fibrotic and osteophytic stabilization of the segment occurs. This phase is associated with fibrosis within the intervertebral joint, enlargement and locking of the facets and periarticular fibrosis. It is also associated with loss of nuclear material within the disc and peripheral osteophyte formation. These changes result in increasing stiffness of the joint .

Causes of the spinal instability:

A, degenerative diseases,

$\mathrm{B}$, postoperative status,

C, trauma to the spine or it's surrounding structures,

D, Development disorders like scoliosis and other congenital spine lesions \&

E, infection.

\section{Radiological Evaluation Of Lumbar Instability}

The diagnosis of vertebral instability is commonly based on the imaging finding of abnormal vertebral motion. There may be abnormal translation and/or rotation around the $\mathrm{x}-, \mathrm{y}-$, and $\mathrm{z}$-axes of the threedimensional coordinates system proposed by Panjabi and White ${ }^{[8] .}$ In this system, the $\mathrm{x}$-axis is horizontal in the coronal plane, from left to right, the y-axis is vertical, or craniocaudal, and the z-axis is horizontal in the sagittal plane, from front to back. Vertebral instability is generally multidirectional, whereas the resulting displacement is evaluated in one plane at a time. Sagittal (front to back, or $\mathrm{z}$-axis) and coronal (side to side, or $\mathrm{x}$-axis) displacements are evaluated on radiographs, and displacements on the axial plane are evaluated on computed tomographic (CT) or magnetic resonance (MR) images.

\section{Structural Changes on Neutral Radiographs}

Several radiographic findings have been proposed as indicators of vertebral instability. To our knowledge, Knuttson ${ }^{[9]}$ was the first to report the vacuum phenomenon in the intervertebral disk and to present its association with lumbar spine instability. Because instability may create excessive intervertebral distraction and subsequent negative intradiskal pressure, allowing interstitial nitrogen in the surrounding tissues to become gaseous and to accumulate within clefts of the degenerated disk, it is assumed that the vacuum phenomenon is often associated with vertebral instability. Moderate disk degeneration with mild disk space narrowing and osteosclerosis also have been associated with vertebral instability. In contrast, a marked disk space narrowing has been considered to be indicative of the late stabilization phase described by Kirkaldy-Willis ${ }^{[6]}$ and Farfan.

Another classic indirect radiographic sign associated with instability is the traction spur, which is located 2 or $3 \mathrm{~mm}$ from the endplate and has a horizontal orientation. The proposed mechanism is that the traction spur is caused by increased tensile stresses exerted by the Sharpey fibers or by those of the anterior longitudinal ligament on the periosteum of the vertebral body, in the case of spinal instability. The claw osteophyte is a bony outgrowth arising very close to the margin of the intervertebral disk, from the vertebral body apophysis, directed with a sweeping configuration toward the corresponding part of the vertebral body opposite the disk. The claw osteophyte is not strictly associated with instability; it is regarded as a result of compression and a sign of stability restoration. Traction spurs and claw osteophytes are thought to represent different stages of the same pathologic process and frequently coexist on the same vertebral rim.

MacGibbon ${ }^{[10]}$ and Farfan suggested that elongated L5 transverse processes (those at least as long as the L3 transverse processes) and a deep-seated L5 vertebra (that situated below the intercrestal line) confer stability on the lumbosacral joint and expose the L4-5 joint to rotational stresses. In contrast, when the intercrestal line passes through the L5 vertebra or through the L5-S1 disk and the transverse processes are short, the lumbosacral joint is at risk to strain. However, Frymoyer and Selby ${ }^{[3]}$ reported no relation between disk degeneration and either intercrestal line position or transverse processes length.

\section{Functional Radiography}

Functional radiography in the sagittal plane can be achieved either in flexion and extension or with passive axial traction and compression. In axial traction and compression radiography, lateral radiographs are obtained with the patient in standing position. Axial traction is accomplished by letting the patient hang by his or her hands from a horizontal bar, whereas compression radiography is performed when the patient has sandbags of approximately $30 \%$ of his or her weight on the shoulders. However, Pitkanen ${ }^{[11]}$ et al, comparing traction-compression with flexion-extension lateral views in a group of 306 patients with clinically suspected 
instability, concluded that traction-compression radiographs were of questionable value in the diagnosis of lumbar instability.

Because of its simplicity, low expense, and wide availability, functional flexion-extension radiography is the most thoroughly studied and the most widely used method in the imaging diagnosis of lumbar intervertebral instability. Many surgeons use flexion-extension lateral views to disclose abnormal vertebral motion before deciding on surgical fusion. However, as reported by Nizard et $\mathrm{al}^{\left[{ }^{[12]}\right.}$, this method is challenging and debatable for the following three reasons: (a) Its diagnostic value cannot be determined because of the lack of a nontraumatic and routinely applicable reference standard to define intervertebral instability; $(b)$ its reproducibility is difficult, a slight variation in patient positioning or in the direction of the x-ray beam may result in a 10\%-15\% variation in the range of vertebral displacement ; and $(c)$ the appropriate way to obtain flexion-extension radiographs and the method to measure displacements are still not standardized.

\section{CT Imaging}

CT provides a detailed view of spinal degenerative changes and facet joint orientation. CT can demonstrate underlying predisposing anatomic factors, such as facet joint asymmetry, that may lead to an abnormal axial rotation of a vertebra on the subjacent one (rotatory spondylolisthesis). This results in accelerated stresses and asymmetric disk and facet joint degenerative changes, particularly asymmetric anterior subluxation of the facet joints, unilateral recess stenosis, and a foraminal disk herniation on the side of maximal facet joint subluxation.

Kirkaldy-Willis $^{[6]}$ and Farfan described a technique of functional CT (twist test), in which the CT scan is obtained through the facet joint while the patient twists the torso and the pelvis is tightly strapped to the CT table. The aim of the twist test was to demonstrate increased abnormal motion, such as a gap of the facet joint space or an abnormal motion during rotation of the trunk, not clearly evident at functional radiography. A facet joint shows increased motion when the cartilage space increases on rotation and when the superior articular process on that side is displaced forward to narrow the root canal. The gap may appear as a vacuum phenomenon into the facet joint space during rotation. However, according to Nizard ${ }^{[12]}$ et al, it is not known whether this technique allows the differentiation between normal and unstable spine.

$\mathrm{CT}$ is the procedure of choice to detect a vacuum phenomenon within the degenerating disks or facet joints, although this finding has no known clinical significance. Functional CT may demonstrate what is considered to be an abnormal motion between two vertebrae, but it is unsuitable for large patient series in view of the exposure to ionizing radiation).

\section{MR Imaging}

MR imaging is generally considered to be the most accurate imaging method for diagnosing degenerative abnormalities of the spine, except for the vacuum phenomenon, and is often used as the diagnostic modality of choice for patients with chronic low back pain. Identification of patients with an increased chance of instability on MR images can be clinically relevant and can influence indications for flexion-extension radiography.

Degenerative diskogenic vertebral changes can be noted on endplates bordering the intervertebral disks (Modic types 1-3) ${ }^{[13]}$. The association of vertebral instability with changes in the bone marrow adjacent to the endplates has been discussed, but without consistent results. Modic ${ }^{[13]}$ et al stated that the clinical importance of these changes in the bone marrow is unknown. Lang et al observed bone marrow changes adjacent to the endplates in postoperative instability, but no statistically significant correlation exists between segmental instability and abnormalities of the bone marrow adjacent to the endplates in patients without spinal fusion, as resulted from a study of Bram et al $(P=.26)$. Conversely, Bram et al found a significant association between radiographic instability and traction spurs and between radiographic instability and annular tears.

In their study of patients with chronic low back pain, Aprill and Bogduk ${ }^{[14]}$ first described annular tears as a high-signal-intensity dot on sagittal T2-weighted images. Therefore, flexion-extension radiographs should be considered in patients with annular tears or traction spurs. Unfortunately, additional studies supporting this conclusion are necessary before it can be generally accepted. A high-signal-intensity zone in the posterior annulus fibrosus on sagittal T2-weighted images has been found much too frequently in asymptomatic subjects to be considered a reliable independent diagnostic indicator.

\section{Clinical And Radiologic Considerations}

Clinical criteria for lumbar spine instability have not yet been clearly defined. Recurrent, acute episodes of low back pain produced by mechanical stresses have been considered to be indicative of instability. If a full return from the bent position fails because of a sudden attack of low back pain (ie, instability catch), if a patient is unable to get a raised, straightened leg to move down and suddenly drops the leg due to a sharp pain in the low back (ie, painful catch), and if a patient feels anxiety resulting from a sensation of collapse of the low 
back because of a sudden attack of back pain during movement (ie, apprehension), the patient fulfills the three criteria for instability described by Kotilainen and Valtonen ${ }^{[15]}$. A loss of tone in the legs or in the low back and pelvic region (ie, giving away phenomenon) has also been observed in some patients with lumbar instability. However, these clinical criteria have not been rigorously evaluated.

Overall, the relationship between imaging instability and its symptoms is controversial. Pitkanen ${ }^{[11]}$ et al found poor correlation between clinical signs of lumbar instability and abnormalities found on functional radiographs. Dvorak et al found that the analysis of the segmental motion of the lumbar spine using functional radiographs does not aid in differentiating the underlying pathologic condition of a patient with low back pain. Conversely, Iguchi et al measured sagittal translation and rotation at the L4-5 segment in flexion-extension radiographs of 1090 outpatients with low back and/or leg pain by using a three-landmark measuring method. The symptoms of four groups with and without 3-mm translation and with and without $10^{\circ}$ sagittal rotation were compared for all patients and for 280 agematched patients by using the scoring system proposed by the Japanese Orthopedic Associatio ${ }^{[16]}$ for assessment of surgical treatment of low back pain. This scoring system is based on subjective symptoms and clinical signs; the total score ranges from 0 to 15 , with only a score of 15 representing an asymptomatic patient with no objective signs. Results showed that patients with 3-mm or greater translation had been suffering from low back and/or leg pain the longest and had significantly lower scores than patients with less than 3-mm translation; however, no difference was observed between the groups in terms of sagittal rotation.

Maigne ${ }^{[17]}$ et al studied 42 patients with low back pain that occurred immediately on sitting down and was relieved on standing up by using functional radiographs and found an important association between this symptom and imaging signs of instability (100\% specificity, $31 \%$ sensitivity) or severe anterior loss of disk space in flexion (87\% specificity, 55\% sensitivity).

\section{Conclusion}

Determination of the relationship between imaging instability and its symptoms remains challenging if not impossible. In the case of degenerative spondylolisthesis and concomitant spinal stenosis at the slip level, the clinical pattern includes buttock and leg pain usually associated with low back pain. These symptoms are brought on with walking and are relieved with resting. Spinal stenosis can cause compression of the cauda equina or individual nerve roots. The classic description of neurogenic claudication from spinal stenosis is bilateral radicular pain, disorders of sensory function, and motor deficits.

All of this uncertainty and controversy creates an ethical burden for all doctors from all disciplines involved in the diagnosis and treatment of patients with low back pain who are thought to be suffering from segmental instability. Currently, imaging helps to select those patients who have supportive evidence of a causeand-effect relationship in their spine that shows the degeneration process associated with segmental instability. It is, however, still far from satisfactory, with significant gaps in the knowledge that will formulate a unified concept of this condition. The quantification of normal and abnormal spinal motion is likely to be still dependent on imaging. It is unlikely that any future agreement of definition, clinical syndromes, and therapeutic regimes can be reached if clinically useful measurements are not a fundamental component of the whole concept of instability.

\section{References}

[1]. Sato H, Kikuchi S. The natural history of radiographic instability of the lumbar spine. Spine 1993; 18: 2075-79.

[2]. Panjabi M. The stabilizing system of the spine. Part II : Neutral zone and instability hypothesis. J Spinal Disord 1992; 5: 390-98.

[3]. Stokes I, Frymoyer J. Segmental motion and instability. Spine 1987; 12: 68-91.

[4]. Junghanns H: Spondylolisthesis ohne spalt in zwischengelenstuck. Archiv fur Orthopaedische und Unfall-Chirurgie 29:118-127, 1930 .

[5]. Newman PH, Stone KH (1963) The aetiology of spondylolisthesis. J Bone Joint Surg [Br] 45:39

[6]. Kirkaldy-Willis W. Symposium on instability of the lumbar spine : Introduction. Spine 1985; 10: 254-55.

[7]. Axelsson P, Johnsson R, Strömqvist B (2000) Is there increased intervertebral mobility in isthmic adult spondylolisthesis? A matched comparative study using roentgen stereophotogrammetry. Spine 25:1701-1703.

[8]. White AA, Panjabi MM. Clinical biomechanics of the spine. Philadelphia: JB Lippincott, 1978.

[9]. KNUTSSON,F. The instability associated with disc generation in the lumbar spine. Acta. Radiologica, Vol. $25,1944$.

[10]. MacGibbon B, Farfan HF. A radiologic survey of various configurations of the lumbar spine. Spine 1979; 4: 258-266.

[11]. Pitkanen M, Manninen H, Lindgrer K. Limited usefulness of traction-compression films in the radiolographic diagnosis of lumbar spinal instability : Comparison with flexion-extension films. Spine 1997; 22: 193-97.

[12]. Remy S Nizard, Marc Wybier, Jean-Denis Laredo. Radiologic assessment of lumbar intervertebral instability and degenerative spondylolisthesis. RadiolClin North Am 2001; 39(1): 55-71.

[13]. Modic MT. Modic type 1 and type 2 changes. J Neurosurg Spine. 2007;6:150-151. doi: 10.3171/spi.2007.6.2.150.

[14]. Aprill C, Bogduk N. High intensity zone: a diagnostic sign of painful lumbar disc on magnetic resonance imaging. $B r J$ Radiol 1992; 65:361-369.

[15]. Kotilainen E, Valtonen S, Carlson C-Å (1993) Microsurgical treatment of lumbar disc herniation: follow-up of 237 patients. Acta Neurochir (Wien) 120: 143-149.

[16]. Japanese Orthopaedic Association. Japanese Orthopaedic Association scoring system for cervical spondylotic myelopathy (in Japanese). Nippon Seikeigeka Gakkai Zasshi 1976;50:18-19.

[17]. Maigne R. Low back pain of thoracolumbar origin. Arch Phys Med Rehabil. 1980 Sep;61(9):389-395. 